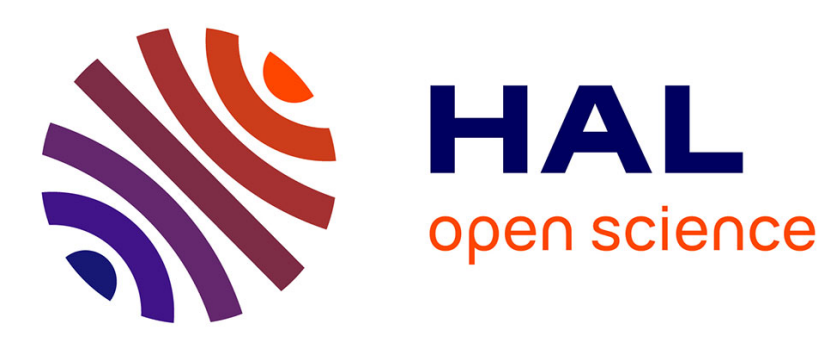

\title{
Vision-based microforce measurement with a large range-to-resolution ratio using a twin-scale pattern
}

Valerian Guelpa, Guillaume J. Laurent, Patrick Sandoz, Cédric Clévy

\section{To cite this version:}

Valerian Guelpa, Guillaume J. Laurent, Patrick Sandoz, Cédric Clévy. Vision-based microforce measurement with a large range-to-resolution ratio using a twin-scale pattern. IEEE/ASME Transactions on Mechatronics, 2015, 20 (6), pp.3148-3156. 10.1109/TMECH.2015.2407053 . hal-01303489

\section{HAL Id: hal-01303489 \\ https://hal.science/hal-01303489}

Submitted on 18 Apr 2016

HAL is a multi-disciplinary open access archive for the deposit and dissemination of scientific research documents, whether they are published or not. The documents may come from teaching and research institutions in France or abroad, or from public or private research centers.
L'archive ouverte pluridisciplinaire HAL, est destinée au dépôt et à la diffusion de documents scientifiques de niveau recherche, publiés ou non, émanant des établissements d'enseignement et de recherche français ou étrangers, des laboratoires publics ou privés. 


\title{
Vision-Based Microforce Measurement with a Large Range-to-Resolution Ratio using a Twin-Scale Pattern
}

\author{
Valérian Guelpa ${ }^{1}$, Guillaume J. Laurent ${ }^{1}$, Patrick Sandoz ${ }^{2}$ and Cédric Clévy ${ }^{1}$ \\ ${ }^{1}$ Automation and Micro-Mechatronics Systems Department, FEMTO-ST Institute, UMR CNRS 6174, ENSMM, \\ Université de Franche-Comté, Besançon, France \\ 2 Applied Mechanics Department, FEMTO-ST Institute, UMR CNRS 6174, ENSMM, Université de \\ Franche-Comté, Besançon, France
}

\begin{abstract}
Force sensors are often required in order to work at the micro-scale but existing ones rarely meet all expectations, particularly in terms of resolution, range, accuracy or integration potential. This paper presents a novel micro-force measurement method by vision, based on a twin-scale pattern fixed on a compliant structure. This approach enabled subpixelic measurement of position by the use of a micro-machined pattern based on Vernier principle. This method also presents flexibility, insensitivity to electronic noise, fast operating time and ease of calibration.

The major contribution consists in the large range-toresolution ratio of the measurement system. With an experimental range of $50 \mathrm{mN}$ and a resolution below $50 \mathrm{nN}$, a range-toresolution ratio of $10^{6}$ is obtained. A repeatability under $7.8 \mu \mathrm{N}$ and a trueness under $15 \mu \mathrm{N}$ have been experimentally measured. Finally, the method can be applied to other specifications and applications in terms of range.
\end{abstract}

Index Terms-Micro-force sensor, vision, twin-scale, range-toresolution ratio

\section{INTRODUCTION}

For some decades many technologies tend toward miniaturization. A lot of improvements passed through the microscale issues in fields such as energy, biology, medicine, instrumentation and robotics. Societal and technological needs strongly push systems to extremely high integration levels (strong desire to have more complex systems with increasing number of functionalities), important miniaturization, smarter and more accurate systems.

Clean room microfabrication technologies and the integration of active materials are notably widespread technologies as well as the use of compliant structures [1]. Despite their strong interest and use (high resolution motion generation, high integration level, accurate fabrication, etc.) several drawbacks still have to be overcome to devise products with expected performances: (i) the behavior of active materials is highly non linear and time varying notably because of strong influence of environmental conditions; (ii) the use of compliant structures amplifies displacements but also non linearities. The integration of sensors to provide closed loop control appears as a relevant solution but several requirements have to be met simultaneously: direct measurement, small size, high bandwidth, high signal to noise ratio, multi degrees-offreedom measurements, etc.
Force measurement is of prime importance to succeed in performing complex tasks such as in microsurgery, biology (characterization of cells, in vitro fertilization) or microassembly ([2], [3], [4]). Intensive researches have been conducted but designed force sensors rarely meet all expectations in terms of range, resolution, accuracy and bandwith; as well as in terms of ease of integration, size, and interactions [5]. This limitation is mainly due to the fact that force measurement cannot be done directly. Either the displacement of a mobile element is measured (in a compliant architecture for example) or a level of stress. Force estimation is then based on a calibration step and on a force-displacement or force-stress (or its derivatives) model. Variations of system behavior and poor signal-to-noise ratio being key features at the microscale, it is then highly important to have the best initial signal quality (displacement or stress). To tackle this key lock many works propose smart, optimized or improved designs and combine it with one measurement principle among the most used: capacitive ([6], [7], [8]), piezoresistive ([9], [10], [11]), strain gauges ([12], [13]), magnetic ( [14], [15]), or whether optical ([16], [10], [17]). Several research teams notably proposed compliant systems with improved shapes and reduced stiffness (notably to improve resolution) ([18], [19]). This choice notably reduces the measurement range of the whole system which may be a great limitation. Most works investigate system designs and enable range-toresolution ratios comprised between $10^{3}$ and $10^{5}$ (see Fig. 1 further).

An alternative approach is to investigate displacements measurement through vision because many progresses have recently been done to provide smaller sensors, with higher image qualities, higher frequencies and because of the extensive use of cameras for microscale applications (cameras are already integrated in many platforms or systems). Among the visual methods some are based on deformation observation of the shape, as Greminger et al. in [20], Wason in [4] or Wang in [21]. Others work on a correlation principle, as Anis et al. in [22] or Chang et al. in [23]. Finally, some works use a special vision method to deal with specific applications, as Karimirad et al. [24] that observe a cell deformation during a simple contact. These works state the interest of measuring 
forces through vision at the microscale but also show related limitations notably because of the severe trade-off between field of observation and achieved resolution but also because of algorithms generally used. Typical range-to-resolution ratios are about $10^{3}$.

To overcome such drawback, we chose in this paper a different approach in the sense that it intends to improve the visual measurement principle by itself. Some visual methods based on pseudo-periodic patterns have been proposed by different authors [25], [26], [27], [28]. These methods allow to extend the measuring range beyond the field-of-view while measuring the pattern position with sub-nanometer resolution. Recently, we proposed a concept based on a twin-scale periodic grid with two different pitch sizes that has also a large range-toresolution ratio and fast operating time [29], [30].

In this paper, we study and demonstrate the potentiality of the combination of this visual method with a compliant structure to achieve force measurements. The principle is based on the observation of the twin-scale pattern fixed on a compliant structure. This novel visual force sensing approach aims at a very high range-to-resolution ratio.

This paper is organized as follows. The measurement principle is presented in section II, the position measurement method and its extension to force measurement are notably introduced. Section III presents the experimental setup. Section IV presents the experimental results and discusses the performances achieved in two steps: firstly the quantification of the method repeatability, resolution and trueness; and secondly the demonstration of the force measurement range. Section $\mathrm{V}$ allows the discussion of these results. Section VI concludes the paper.

\section{PRinciple And potential CAPABilities of the METHOD}

\section{A. Estimation of force through vision}

Forces are extensive physical properties which can not be directly measured. A common way to measure forces is to measure the deformation of a test specimen. For instance, the Hooke's law states that the force $F$ exerted on an ideal spring is proportional to its deformation, i.e.,

$$
F=K \cdot \delta
$$

with $K$ the stiffness of the spring and $\delta$ the relative displacement from its relaxed position.

Measuring micro-scale forces often involves to use a very low stiffness. For example, the typical stiffness of an atomic force microscope is about $0.1 \mathrm{~N} . \mathrm{m}^{-1}$ [31]. Alternative solutions rely on higher stiffness structures (few hundreds of $\mathrm{N} . \mathrm{m}^{-1}$ ) combined with high-sensitive displacement sensors (see an example in [32]).

Unfortunately, obtaining a high resolution in displacement measurement is generally detrimental to the allowed range of measurement. Common values of the range-to-resolution ratio of force sensors are about $10^{3}$, sometimes $10^{4}$ and rarely $10^{5}$ (as illustrated in Fig. 1). ${ }^{1}$

\footnotetext{
${ }^{1}$ The metrologic terms used in this paper are those defined by the Joint Committee for Guides in Metrology [33].
}

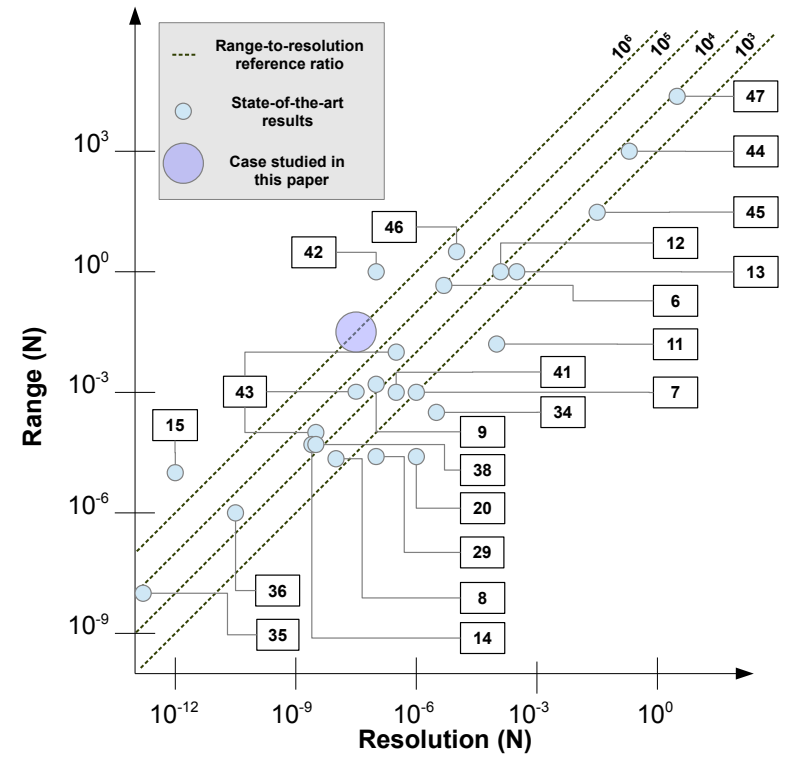

Fig. 1. Expected range-to-resolution performance (dashed line) with regards to state-of-the-art works and commercial force sensors. Numbers correspond to the references of the articles and sensors.

\section{B. Twin-scale vision based measurement}

Recently, we proposed a novel vision-based method that allows displacement measurements with improved range-toresolution ratios. This method is an extension of phase processing methods of periodic image features [48]. Its working principle and the evaluation of these performances are detailed in [30] as well as discussions about the influence of the various parameters. This section summarizes the core content of this article.

The principle of this phase measurement method relies on the phase-to-displacement relationship that is a scalar product in the frequency domain:

$$
\mathcal{F}(f(x-\delta))=e^{-2 \pi i \delta \xi} \cdot \mathcal{F}(f(x))
$$

where $\mathcal{F}$ stands for the Fourier transformation. $f(x)$ is the space function considered (here the intensity of the pixels), $x$ the spatial coordinate, $\delta$ the spatial displacement and $\xi$ the reciprocal variable of $\mathrm{x}$ (or transform variable). A target displacement $\delta$ induces only a phase shift $\Phi$ in the frequency domain with $\Phi=2 \pi \delta \xi$.

Thanks to this linear relationship, we can retrieve object displacements with a very high precision through phase processing of the recorded images. In order to get efficient computation times and high signal-to-noise ratios, a highcontrast periodic-pattern can be placed on the target of interest (see Fig. 2). Then, instead of performing a complete Discrete Fourier Transform (DFT), we can compute a single-frequency spectral component; that corresponds to the spatial frequency of the periodic pattern used. In the present case, a complex analysis vector $Z(k)$ defined by a Gaussian window and a periodic signal at the period $L$ of the stripe set is used:

$$
Z(k)=e^{-\left(\frac{k-N / 2}{N / 4.5}\right)^{2}} \cdot e^{-\left(\frac{2 i \pi(k-N / 2)}{L}\right)}
$$




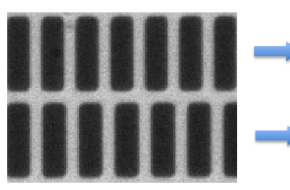

Twin stripe pattern

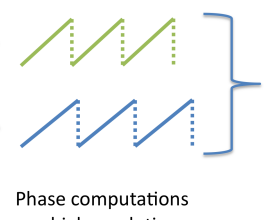

=> high resolution

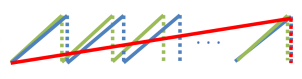

Removal of phase ambiguities $\Rightarrow$ extended range
Fig. 2. Working principle of the twin-scale visual measurement. See [30].

where $k$ is the pixel index and $N$ is the image width in pixels.

The expected phase $\Phi$ is then given by the argument of the dot product between vector $Z$ and the vector of pixel intensities. The target displacement can then be determined by:

$$
\delta=\frac{\Phi \cdot L}{2 \pi}+n L
$$

where $n$ is an unknown integer standing for an entire number of stripe periods. Indeed due to the stripe periodicity, the displacement value is obtained modulo $L$ since different positions distant from an entire number of periods produce indistinguishable images.

Our method to overcome this limitation consists in the use of a twin-scale. The use of a second stripe set with a slightly different period provides complementary and independent phase data that can be used for the removal of phase ambiguities. The principle is illustrated in Fig. 2. Thanks to the progressive mismatch between the two stripe sets, phase ambiguities can be removed and the unambiguous range switches from a single period to a new value $\Lambda$ given by:

$$
\Lambda=\frac{L_{1} \cdot L_{2}}{\left|L_{1}-L_{2}\right|}
$$

where $L_{1}$ and $L_{2}$ are the twin-stripe set periods.

The last step consists in using synthetic data to determine the correct $2 m \pi$ constant to apply to the phase shift observed for either stripe set:

$$
\delta=\frac{\Phi_{1} \cdot L_{1}}{2 \pi}+m L_{1}+p \Lambda
$$

in which $\Phi_{1}$ is the phase shift for the smallest stripe set, $m$ the number of periods derived from the synthetic phase and $p$ an unknown number of periods $\Lambda$ that represents the new ambiguity range. In practice, if the range of displacement is below $\Lambda, p$ does not change and can be set arbitrary to 0 . It is important to mention that the actual periods $L_{1}$ and $L_{2}$ of the patterns are accurately known, so the pattern features provide a known size reference in images allowing a direct conversion from pixels to meters. The method is thus self-calibrating concerning the position measurement and does not depend on experimental parameters such as magnification or field of view. Moreover the process of phase measurement induces a large spectral filtering, that makes it possible to get away from problems of dynamic range and high-frequency spatial noises. As described in [49], the image processing technique presents also a low sensitivity to the recorded image sharpness as long as the spatial frequencies associated to the twin-scale patterns
TABLE I

PERFORMANCES OF TWIN-SCALE VISUAL MEASUREMENT WITH A $8 \mu \mathrm{M}$ PERIODIC PATTERN AND 640X480 8-BITS CAMERA (FROM [30]).

\begin{tabular}{lc}
\hline Property & Value \\
\hline Travel range & $168 \mu \mathrm{m}$ \\
Resolution & $55 \mathrm{pm}$ \\
Experimental repeatability $(3-\sigma)$ & $5 \mathrm{~nm}$ \\
Bandwidth & $>1500 \mathrm{~Hz}$ \\
\hline
\end{tabular}

are imaged with a sufficient contrast. Therefore the resolution of the microscope is not a critical parameter.

Table I gathers the performances of the method using a micro-machined pattern with $8 \mu \mathrm{m}$ and $8.4 \mu \mathrm{m}$ twin scales periods, corresponding to an unambiguous range of $168 \mu \mathrm{m}$. The range-to-resolution ratio of the displacement measurement method is $3 \cdot 10^{6}$ with a 8 -bits camera [30].

Applying this method to measure the displacement of an elastic structure appears as very promising to design force sensors with large range-to-resolution ratios.

\section{Potentiality in force measurement}

A twin-scale pattern is fixed onto a compliant structure whose stiffness is known. The idea is to estimate the force applied to it by measuring its displacement. For example, using the $8 / 8.4 \mu \mathrm{m}$ pattern attached to a compliant structure with a stiffness of $1000 \mathrm{~N} . \mathrm{m}^{-1}$ may lead to a force sensor able to measure up to $168 \mathrm{mN}$ with a resolution of $55 \mathrm{nN}$ in theory (performances deduced from Table I). Thus, the potential range-to-resolution ratio of such a sensor could be $3.10^{6}$.

Comparing these expected performances with state-of-theart works and commercial force sensors, the proposed method could constitute an improvement of at least one order of magnitude in term of range-to-resolution ratio. Fig. 1 allows for a comparison between the presented method and different sensor principles (optical, capacitive, piezo...). The placement criteria are range and resolution. The larger circle represents the experimental performances of the method, investigated latter with the use of a basic 8-bits camera. Other performances (for example repeatability and signal-to-noise ratio) depend on experimental conditions and are not processed in this graph.

This figure illustrates that we can expect better than most of the state-of-the-art sensor performances. Moreover according to the desired range and resolution in the force domain, a couple stiffness/size can be chosen to cover the desired specifications. Thus the sensor could be adapted to many scales, from the micro-scale to the macro-scale as illustrated in Fig. 3. It is important to notice that such scale-adjustments don't affect the range-to-resolution ratio. Nevertheless, the range-to-resolution ratio could be increased by the use of a more efficient camera.

\section{EXPERIMENTAL SETUP AND STIFFNESS DETERMINATION}

To validate experimentally this potential, we use a setup built around a compliant structure on which a pattern is fixed. 


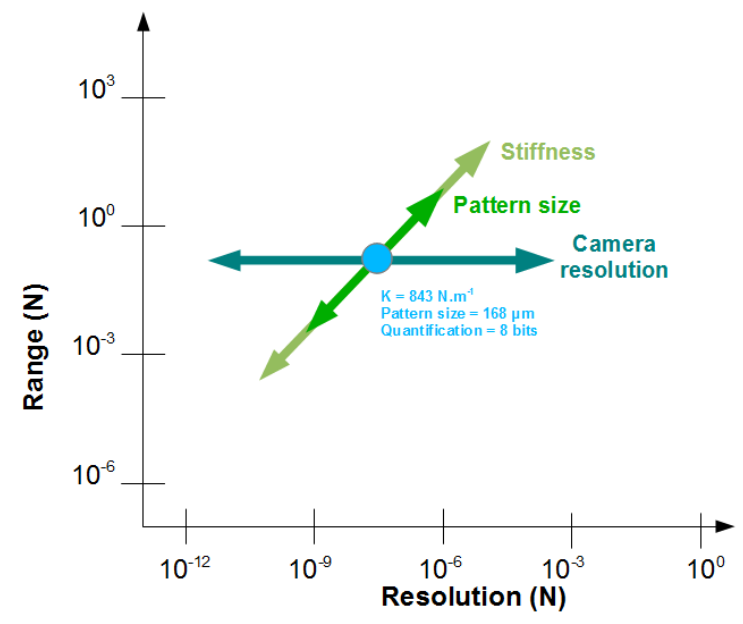

Fig. 3. Graphical representation of the potential evolutions in term of range-to-resolution of the presented method (we assume that environmental disturbances are neglected).

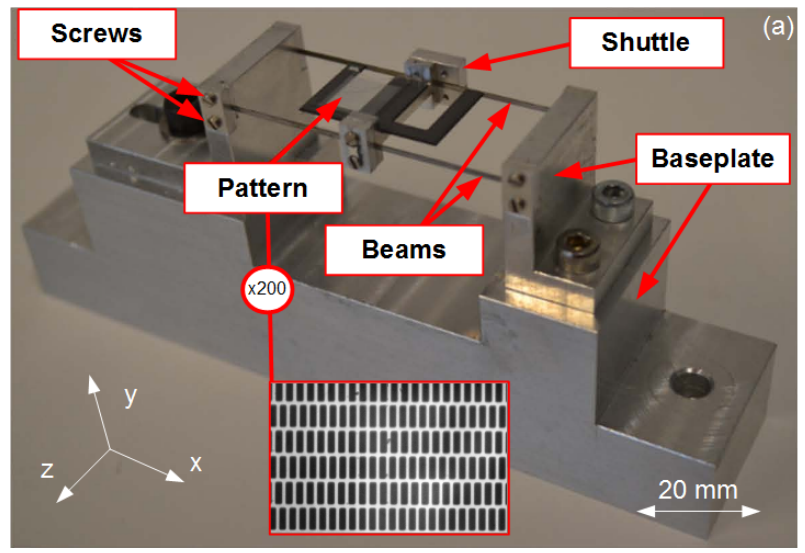

(b)

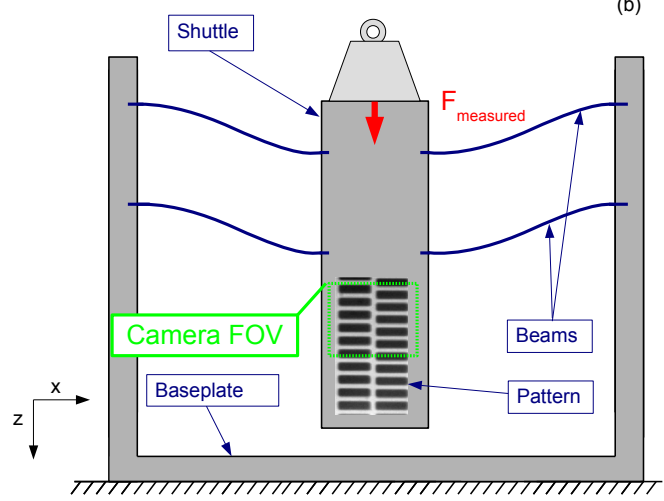

Fig. 4. The compliant structure on which a pattern is attached. The link between the shuttle and the baseplate is four beams allowing the translation of the shuttle. The camera's field of view (FOV) is focused on the pattern. (a) Photo of the compliant setup. The insert shows a typical recorded image. (b) Schema of its operating principle.

In addition to that, a reference sensor that allows the validation of our measurements is used.

\section{A. Force measurement device}

The compliant structure is presented in Fig. 4. It is composed of a mobile shuttle connected to its baseplate by four thin compliant beams, restricting the motions along a single axis (z). The principle of this setup is extracted from [50]. From the knowledge of the stiffness between the shuttle and the baseplate, we derive the forces applied to the shuttle from the measurement of its displacements. On this compliant structure, we attached a twin-scale pattern on the shuttle in order to measure its position and displacements along the $\mathrm{z}$ axis.

The beam stiffness notably depends on the screws clamping the beams on the baseplate (see Fig. 4(a)). The screws allow adding a preload in the beams. This preload increases the stress in the beam and thus the stiffness of the compliant structure. Experiments show that the stiffness can be tuned from 200 to $1000 \mathrm{~N} \cdot \mathrm{m}^{-1}$. This flexibility allows to adapt the measurement range to the application. It was used in the experiments to be as close as possible to the reference sensor range.

The visual setup is composed of a FireWire camera (Allied Vision Technology Pike F-032B, 8 bits, $640 \times 480$ pixels) equipped with a $10 \times$ microscope lens. The computer used to process images is a common computer. The different elements of the setup are fixed on an antivibration table, a paramount element to minimize the impact of mechanical noises.

\section{B. Stiffness measurement procedure}

As mentioned above, the stiffness of the structure has to be known accurately to derive forces from measured displacements. At this stage, the use of a calibration method is mandatory.

The compliant structure stiffness $K$ is measured from the processing of the free oscillations of the shuttle in response to a starting pulse:

$$
w_{0}=\sqrt{\frac{K}{m}}
$$

where $m$ is the mass of the mobile part and $w_{0}$ its natural frequency. Fig. 5 gives an example of free oscillations as reconstructed by the displacement measurement method used.

The stiffness can thus be derived from the free oscillation frequency. The unknown mass $m$ is determined by repeating this recording after the introduction of an additional mass $m_{\delta}$; i.e. once unladen (mass $m$ ) and once with an additional calibrated mass $m_{\delta}$ laid on the shuttle:

$$
m=\frac{m_{\delta} \cdot w_{0, m+m_{\delta}}^{2}}{w_{0, m}^{2}-w_{0, m+m_{\delta}}^{2}}
$$

Beforehand, the mass $m_{\delta}$ is measured accurately. Then the mass $m$ of the mobile part can be deduced, and therefore the stiffness $K$. In this way we dispose of a fast and easy stiffness calibration procedure.

By way of an example, an additional mass $m_{\delta}=1.107 \mathrm{~g}$ was used to do the calibration. This value was measured with a calibrated balance (resolution : $10^{-4} \mathrm{~g}$ ). We observed $w_{0, m}=$ $318.9 \mathrm{~s}^{-1}$ and $w_{0, m+m_{\delta}}=268.2 \mathrm{~s}^{-1}$. Finally, the mass of the shuttle is identified as $m=2.673 \mathrm{~g}$, which allows to calculate the stiffness $K=271.9 \mathrm{~N} . \mathrm{m}^{-1}$. From that moment it's faster to re-calibrate the system because the mass $m$ is known, so we only need to measure $w_{0, m}$ to know the stiffness. 


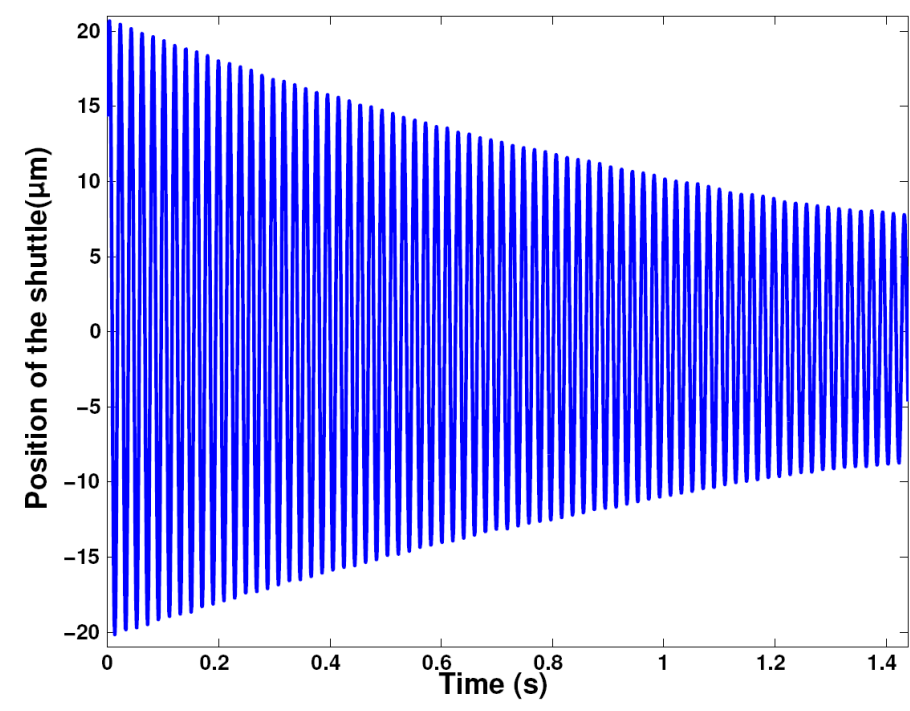

Fig. 5. Free shuttle oscillations in response to a starting pulse as measured by vision at $1389.5 \mathrm{fps}$.

The accuracy of the stiffness evaluation is dependent on the quality of additional mass characterization and on frequency measurement. However the following experiments illustrate the quality of the calibration method.

\section{EXPERIMENTAL PERFORMANCE EVALUATION}

The performances of the proposed force sensing system were experimentally quantified in two steps. The first one, presented in section "Microscale experiment", evaluates repeatability, resolution and trueness. The second one focuses on sensing range, and is developed in "Large range experiment".

\section{A. Microscale experiment}

In order to determine the precision of the method (with repeatability, resolution and trueness), we realized measurements using a FemtoTools microforce sensing probe (model FT-S270) as reference sensor (see Fig. 6). This capacitive sensor has a force range of $2 \mathrm{mN}$ and a resolution of $0.4 \mu \mathrm{N}$ (datasheet). Moreover we corrected its non-linearity with its calibration data (full curve provided by the manufacturer).

The sensor is attached to a nanopositioning XY stage (Piezosystem Jena PXY 200 D12) with a $200 \mu \mathrm{m}$ displacement range and integrated capacitive sensors with a $0.4 \mathrm{~nm}$ resolution. To reduce environment noises, the setup was located in a room that is controlled in temperature $\left(\Delta T<1^{\circ} \mathrm{C}\right)$, in humidity, and mechanically isolated from the rest of the building to minimize mechanical disturbances.

The experiment consists in the observation of 50 sinusoidal cycles performed by the nanopositioning stage along the $\mathrm{z}$ axis. The stage pushes on the shuttle via the reference sensor. 150 measurements (20 s per cycle) are realized during each period of the sinusoid. The stiffness of the compliant structure during this experiment is set as $240 \mathrm{~N} . \mathrm{m}^{-1}$ : a low stiffness allows to measure little forces for a corresponding large displacement.

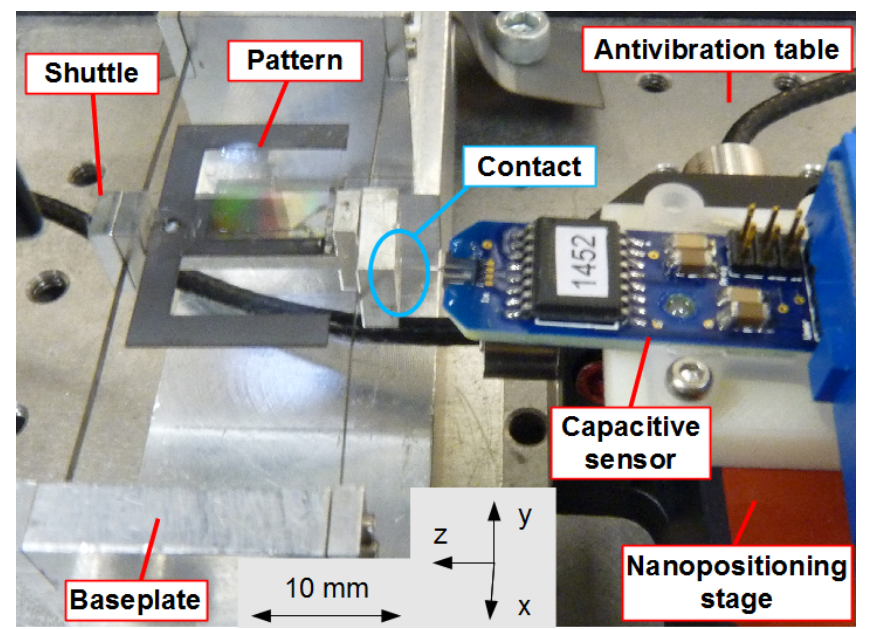

Fig. 6. Experimental setup used for the evaluation of the repeatability. A motorized nanopositioning stage is used to move the reference sensor along $\mathrm{z}$ relatively to the mobile shuttle.

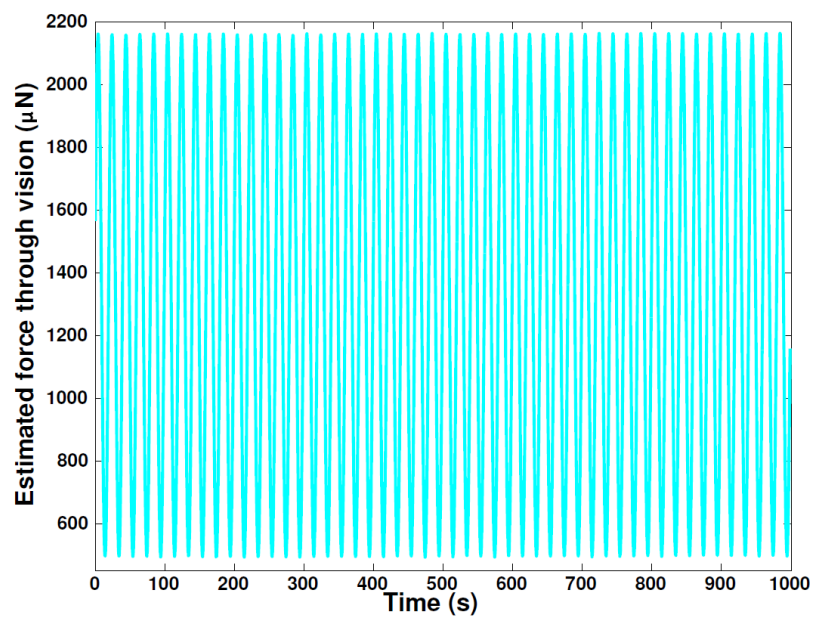

Fig. 7. Experimental force measurement vs time for sinusoidal motions of the nanopositioning stage.

Fig. 7 illustrates the experimental data measured by the visual method during the 50 cycles. Fig. 8 presents the average measurement of the sinusoid, each point corresponding to the average of 50 points of the Fig. 7.

We can extract from this experiment the repeatability of the force estimation, defined as three times the standard deviation of the measured force. The repeatability is presented in the zoom of Fig. 8. We obtain here a $3 \sigma$ repeatability of $7.8 \mu \mathrm{N}$.

However this result notably includes the unknown repeatability of the nanopositioning stage. As an estimation, a nanoactuator has a repeatability about 100 times bigger than its resolution (see [51]). The resolution of this stage is $0.4 \mathrm{~nm}$, so its repeatability could be evaluated to $40 \mathrm{~nm}$. Multiplying by the stiffness of our device (240 N.m ${ }^{-1}$ ), an estimation of the repeatability of the positioning stage in terms of applied force can be $9.6 \mu \mathrm{N}$. This estimated repeatability and the measured repeatability are of the same order of magnitude. We can thus conclude that the repeatability of our visual method is likely intrinsically better than the value observed experimentally; i.e. 


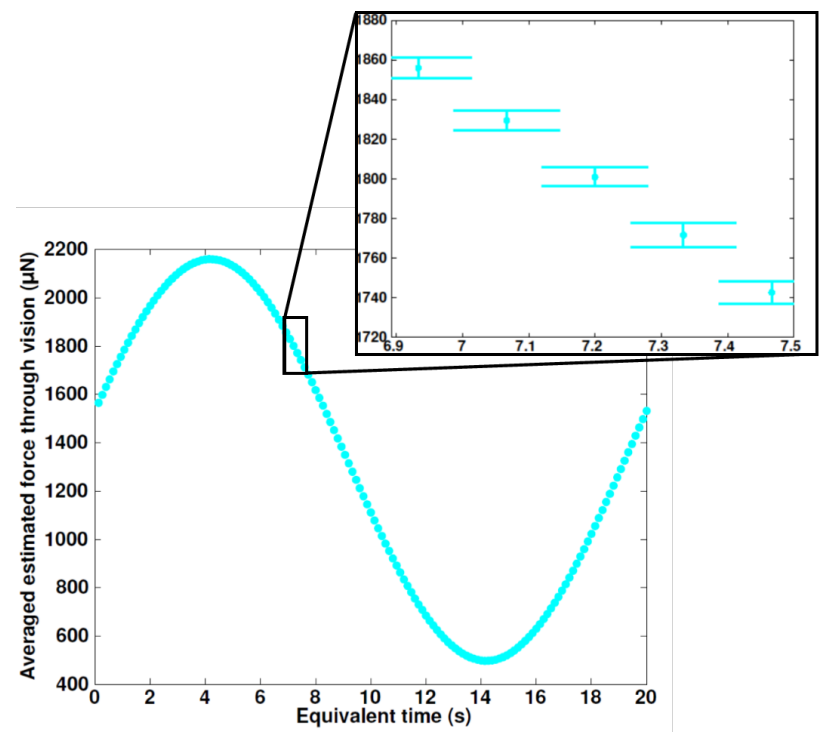

Fig. 8. Average of the force measurement during the sinusoidal displacement. The zoom presents the repeatability derived from the standard deviation of these values $(3 \sigma)$.

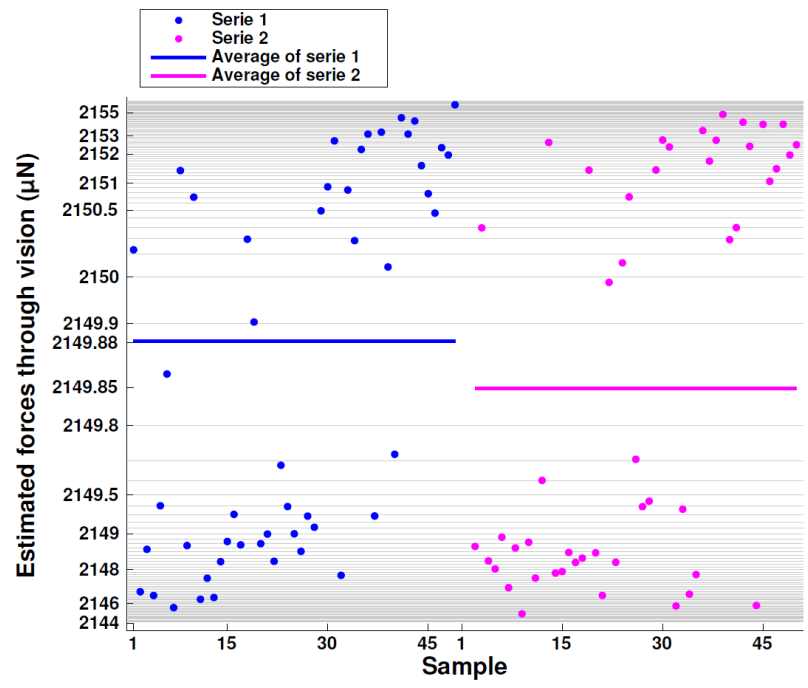

Fig. 9. Two series of measured forces, acquired respectively to an equivalent time of $3.74 \mathrm{~s}$ and $4.66 \mathrm{~s}$ (see Fig. 8). The difference between the average of the two series is the resolution, equal to $32.6 \mathrm{nN}$. The low point density in the central part is due to the non-linear scale allowing the emphasizing of the difference between the averaged values obtained.

\section{$7.8 \mu \mathrm{N}$.}

The second characteristic that we can extract from this experiment is the resolution. The resolution is defined as "smallest change in a quantity being measured that causes a perceptible change in the corresponding indication".

We calculated the averaged sinusoid (see Fig. 8), that allows to filter out an important part of the noise. The smallest difference between two points of this curve is of $32.6 \mathrm{nN}$ (see Fig. 9), corresponding to a small but actual displacement. This little force difference is the experimental resolution, near to the theoretical resolution (given in [30]) here equal to $13.2 \mathrm{nN}$ for a stiffness equal to $240 \mathrm{~N} \cdot \mathrm{m}^{-1}$.

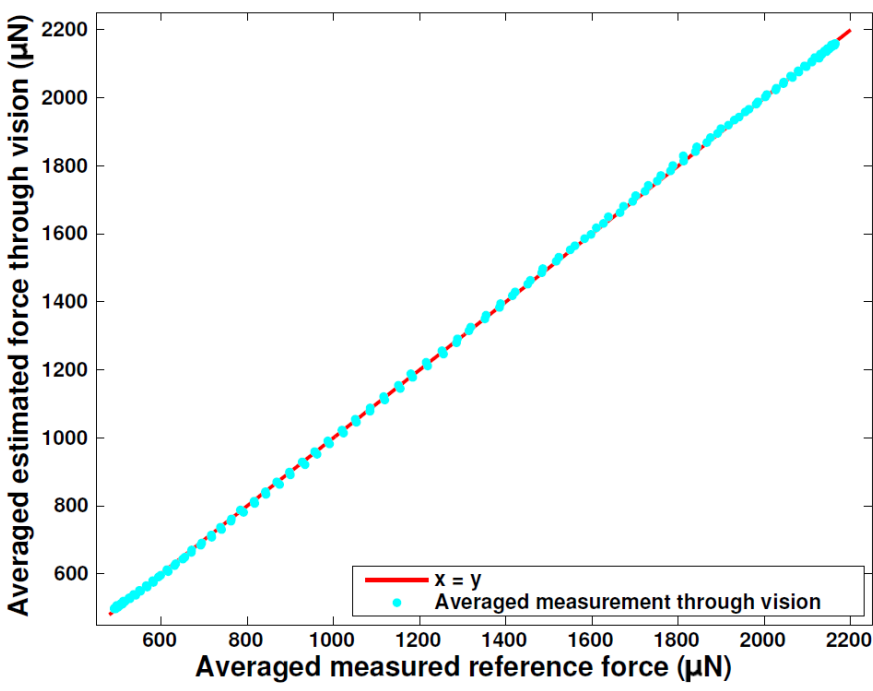

Fig. 10. Averaged force measurements versus averaged reference measurements. Each point corresponds to the average of 50 measures (for both visual and reference sensors). Forces applied were chosen in the working range of the reference sensor, i.e. $[0 ; 2.2 \mathrm{mN}]$.

In a last step, we compared the visual and reference sensor measurements to evaluate the method's trueness, defined as the "closeness of agreement between the average of an infinite number of replicate measured quantity values and a reference quantity value" [33]. We consider a large number of measures to evaluate it. For that, we compare the averaged value of the visual force measurements with the average value provided by the reference sensor. The resulting data are plotted in Fig. 10 that shows good agreement and linearity between the visual and reference measurement tools. The error between the two curves is always inferior to $15 \mu \mathrm{N}$. This point allows to say that the trueness between the visual measurement and the reference sensor is $15 \mu \mathrm{N}$. Moreover this good agreement between the two curves validates the calibration approach (cf. sect. III-B) that doesn't require any reference force sensor.

These experimental results demonstrate that the proposed visual method allows reliable force measurements with a repeatability under $7.8 \mu \mathrm{N}$, a resolution below $32.6 \mathrm{nN}$ and a trueness below $15 \mu \mathrm{N}$.

\section{B. Large range experiment}

A second experimental setup (see Fig. 11) has been used to perform large range experiments, to quantify the range of the sensor. The vision-based force measurements are compared to those provided by a reference sensor: a calibrated balance (Mettler Toledo ML3002: repeatability $\sigma=0.01 \mathrm{~g} \simeq 100 \mu \mathrm{N}$; linearity $0.02 \mathrm{~g} \simeq 200 \mu \mathrm{N}$ ). The compliant structure is attached on a micro-positioning table above this balance and is moved along $\mathrm{z}$ axis. In this way the shuttle applies a force on the balance when moved vertically. At equilibrium, the force applied to the balance is equal to that supported by the shuttle. In this experiment, the stiffness was evaluated equal to $843 \mathrm{~N} \cdot \mathrm{m}^{-1}$. 


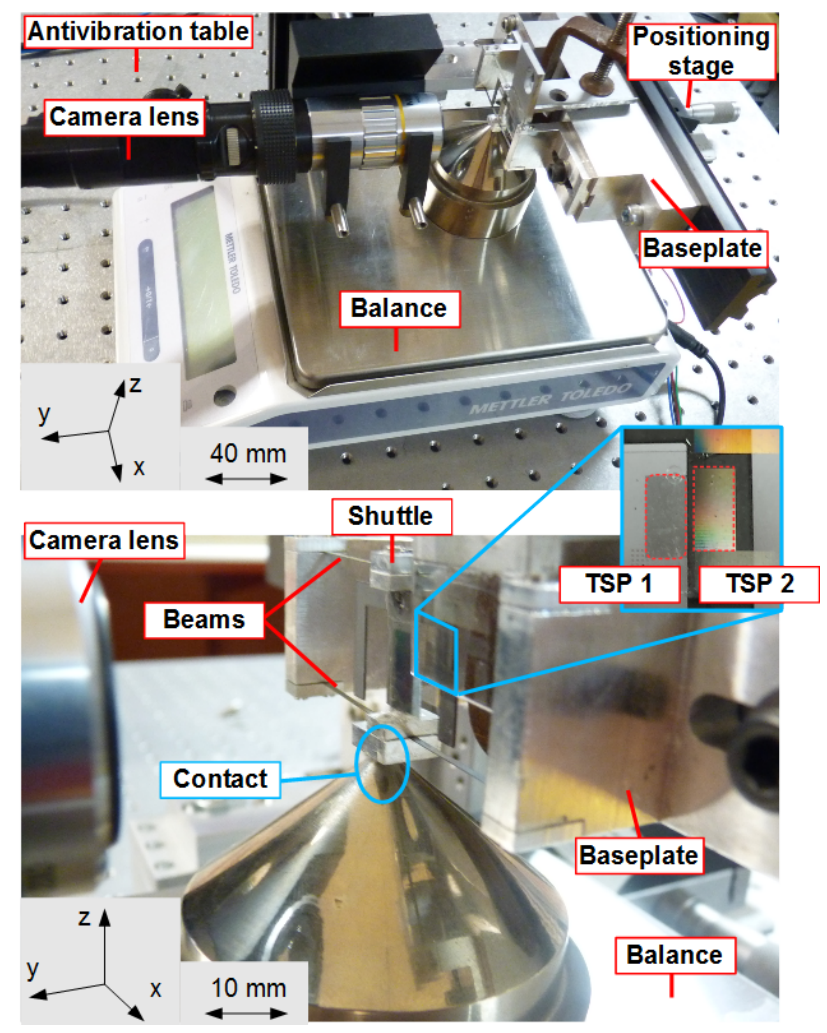

Fig. 11. Experimental setup used for quantification of the measurement range of the sensor. The compliant structure is the one presented in Fig. 4. The shuttle applies a force on the balance when displaced by means of the micro-positioning table. Two twin-scale patterns (TSP) are used: one fixed on the shuttle; the other one fixed on the baseplate. The camera's FOV is focused on the two twin-scale patterns to provide a differential measurement of the displacement proportional to the force.

To measure the shuttle displacement relatively to its baseplate, two twin-scale patterns were used: a first one fixed on the shuttle, the other one on the baseplate (respectively patterns 1 and 2 on Fig. 11). As the baseplate is moving with regards to the camera, such a differential measurement is necessary and the expected force is then proportional to the difference between the baseplate and shuttle displacements; that is also given by the difference between the positions of the two patterns.

Firstly a null force is measured (without contact) to correlate the visual sensor and the reference sensor. Then forces were randomly applied between $19.2 \mathrm{mN}$ and $50.2 \mathrm{mN}$. Fig. 12 presents the visual measurement of the force and that provided by the balance, according to the balance measurement.

Fig. 13 illustrates the deviation between the two force measurements. For a force range of $50.2 \mathrm{mN}$ the peackto-peack error is $303 \mu \mathrm{N}$, so a linearity equals to (303. $\left.10^{-6}\right) /\left(50.2 \cdot 10^{-3}\right)=0.6 \%$ is measured.

\section{DISCUSSION}

The experiments have demonstrated a linear range of $50.2 \mathrm{mN}$ and a resolution of $32.6 \mathrm{nN}$. A straightforward calculus of the range-to-resolution ratio gives $1.5 \cdot 10^{6}$. On closer examination, things are a bit more complicated because

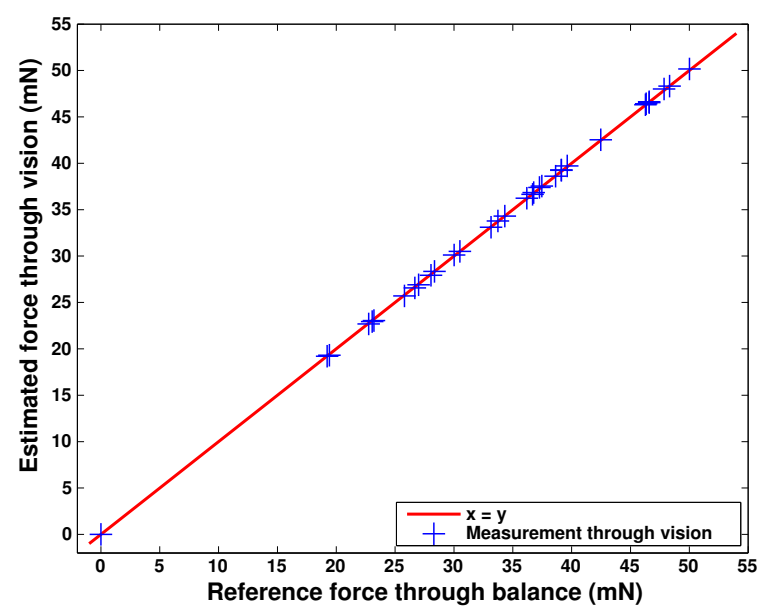

Fig. 12. Visual force estimation through vision compared with the balance measurements, used as reference sensor. Forces are applied by random vertical displacements of the compliant structure, which presses on the balance.

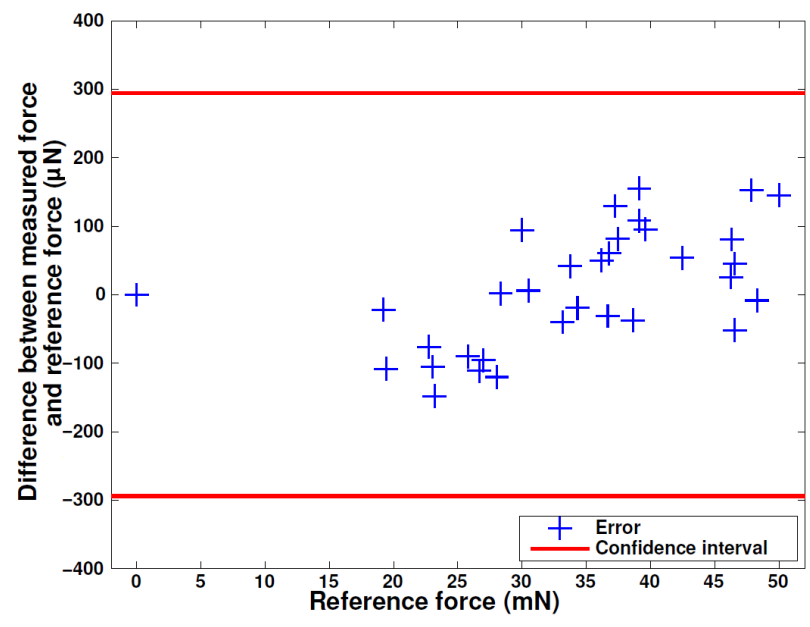

Fig. 13. Deviation between visual and balance measurements. Results remain within the balance confidence interval as defined by its repeatability and linearity.

the stiffness of the compliant structure was different for both experiments.

In the range experiments, the stiffness was $843 \mathrm{~N} . \mathrm{m}^{-1}$. So, when a force of $50.2 \mathrm{mN}$ is applied, the displacement is $59.5 \mu \mathrm{m}$. In the resolution experiments, stiffness was $240 \mathrm{~N} . \mathrm{m}^{-1}$. The force required to get a displacement of $59.5 \mu \mathrm{m}$ is then $14.3 \mathrm{mN}$. In this case, the range-to-resolution ratio is $4.4 \cdot 10^{5}$.

Nevertheless, only a part of the full displacement range of the visual method is used. Indeed beyond $60 \mu \mathrm{m}$ of deformation the force is not proportional to the displacement anymore. Anyway it is possible to increase the measurement range of forces using a non-linear model in place of the Hooke's law. As the unambiguous displacement range is $168 \mu \mathrm{m}$, forces up to $40.3 \mathrm{mN}$ could be measured with a stiffness of $240 \mathrm{~N} . \mathrm{m}^{-1}$ leading to a range-to-resolution ratio of $1.2 \cdot 10^{6}$.

The experimental range-to-resolution ratio is below the 
expected one calculated in theory but in same the order of magnitude, i.e. $10^{6}$.

\section{CONCLUSION}

The presented method meets many expectations raised by the use of force sensors, notably at the micro-scale. The experiments with a calibrated balance have demonstrated a range of $50.2 \mathrm{mN}$ with a linearity below $0.6 \%$. The repeatability of the measurement has been evaluated to $7.8 \mu \mathrm{N}$ and the resolution obtained experimentally is below $32.6 \mathrm{nN}$. The comparison of the measurements with a calibrated reference force sensor showed that the trueness is $15 \mu \mathrm{N}$.

These performances are at the cutting edge of the state of the art in term of range-to-resolution ratio which reaches the order of magnitude of $10^{6}$. Beyond these figured performances, the method shows also a flexibility welcomed at micro-scale: remote measurement, insensitivity to electronic noise, and camera self-calibration.

Further works on the design of the compliant structure would enable to further improve the performances. For instance a monolithic structure could reduce the sensitivity to the perturbations and thus improve the repeatability and the trueness. The linear part of the sensor could also be enlarged with a better design. Finally, a more effective camera would improve a lot the resolution: the use of a 12-bits camera instead of 8-bits would for example divide by 16 the theoretical resolution. All these improvements could lead together to gain another order of magnitude in the range-to-resolution ratio.

\section{ACKNOWLEDGEMENT}

This work was supported by the Smart Blocks project (ANR-251-2011-BS03-005), by Labex ACTION project (ANR-11-LABX-0001-01) and by Région de Franche-Comté. Authors acknowledge the French RENATECH network through its FEMTO-ST technological facility MIMENTO. Authors also acknowledge Micky Rakotondrabe for his technical support.

\section{REFERENCES}

[1] Y. Li and Q. Xu. Design and Robust Repetitive Control of a New Parallel-Kinematic XY Piezostage for Micro/Nanomanipulation. IEEE Transactions on Mechatronics, 17(6):1120-1132, 2012.

[2] M. Rakotondrabe, A. Ivan, S. Khadraoui, P. Lutz, and N. Chaillet. Simultaneous displacement and force self-sensing in piezoelectric actuators and applications to robust control of the displacement. IEEE Transactions on Mechatronics, 20(6):1-13, 2014.

[3] K. Rabenorosoa, C. Clevy, Q. Chen, and P. Lutz. Study of Forces during Micro-Assembly Tasks using Two-Sensing-Fingers Gripper. IEEE Transactions on Mechatronics, 17:811-821, 2012.

[4] J. D. Wason, J. T. Wen, J. J. Gorman, and N. G. Dagalakis. Automated multiprobe microassembly using vision feedback. IEEE Transactions on Robotics, 28(6):1090-1103, 2012.

[5] C. Clevy, M. Rakotondrabe, and N. Chaillet. Signal Measurement and Estimation Techniques for Micro and Nanotechnology. Springer, 2011.

[6] R. J. Wood, K-J Cho, and K. Hoffman. A Novel Multi-Axis Force Sensor for Microrobotics Applications. Smart Materials and Structures, 18, 2009.

[7] F. Beyeler, S. Muntwyler, and B. J. Nelson. A Six-Axis MEMS Force and Torque Sensor With Micro-Newton and Nano-Newtonmeter Resolution. Journal of Microelectromecanical Systems, 18(2):433-441, 2009.
[8] Y. Sun, S. N. Fry, D. P. Potasek, D. J. Bell, and B. J. Nelson. Characterizing Fruit Fly Flight Behavior Using a Microforce Sensor With a New Comb-Drive Configuration. Journal of Microelectromechanical Systems, 14(1):4-11, 2005.

[9] B. Komati, J. Agnus, C. Clevy, and P. Lutz. Prototyping of a Highly Performant and Integrated Piezoresistive Force Sensor for Microscale Applications. Journal of Micromechanics and Microengineering, 24 2014.

[10] M. Dienwiebel, E. De Kuyper, L. Crama, and J. W. M. Frenken. Design and Performance of a High-Resolution Frictional Force Microscope with Quantitative Three-Dimensional Force Sensitivity. Review of Scientific Instrument, 76(4):043704, 2005.

[11] P. Estevez, J. Bank, M. Porta, J. Wei, P.M. Sarro, M. Tichem, and U. Staufer. 6 DOF Force and Torque Sensor for Micro-Manipulation Applications. Procedia Engineering, 25:39-42, 2011.

[12] K. Kristiansen, P. McGuiggan, G. Carver, C. Meinhart, and J. Israelachvili. 3D Force and Displacement Sensor for SFA and AFM Measurements. Langmuir, 24:1541-1549, 2008.

[13] P. J. Berkelman, L. L. Whitcomb, R. H. Taylor, and P. Jensen. A Miniature Microsurgical Instrument Tip Force Sensor for Enhanced Force Feedback During Robot-Assisted Manipulation. IEEE Transaction on Robotics and Automation, 19(5):917-922, 2003.

[14] J. Abadie, E. Piat, S. OSter, and M. Boukallel. Modeling and Experimentation of a Passive Low Frequency Nanoforce Sensor Based on Diamagnetic Levitation. Sensors and Actuators A: Physical, 173:227237, 2012

[15] V. Nesterov. Facility and Methods for the Measurement of Micro and Nano Forces in the Range Below $10^{5} \mathrm{~N}$ with a Resolution of $10^{12} \mathrm{~N}$ (Development Concept). Measurement Science and Technology, 18:360366, 2007.

[16] X. J. Zhang, S. Zappe, R. W. Bernstein, O. Sahin, C. C. Chen, M. Fish, M. P. Scott, and O. Solgaard. Micromachined Silicon Force Sensor Based on Diffractive Optical Encoders for Characterization of Microinjection. Sensors and Actuators A: Physical, 2004.

[17] Y. Zhou, N. Bradley, and V. Barmeshwar. Integrating Optical Force Sensing with Visual Servoing for Microassembly. Journal of Intelligent and Robotic Systems, 28:259-276, 2000.

[18] A. N. Reddy, N. Maheshwari, D. K. Sahu, and G. K. Ananthasuresh. Miniature compliant grippers with vision-based force sensing. IEEE Transactions on Robotics, 26:867-877, 2010.

[19] D. J. Cappelleri, G. Piazza, and V. Kumar. A two Dimensional Vision-Based Force Sensor for Microrobotic Applications. Sensors and Actuators A: Physical, 171:340-351, 2011.

[20] M. A. Greminger and B. J. Nelson. Vision-Based Force Measurement. IEEE Transaction on Pattern Analysis and Machine Intelligence, 26(3):290-298, 2004.

[21] X. Wang, G. K. Ananthasuresh, and J.P. Ostrowski. Vision-Based Sensing of Forces in Elastic Objects. Sensors and Actuators A: Physical, 94:142-156, 2001.

[22] W.L. Cleghorn Y.H. Anis, J.K. Mills. Vision-based measurement of microassembly forces. Journal Of Micromechanics and Microengineering, 16:1639-1652, 2006.

[23] R. J. Chang, C. C. Shiu, and Cheng C. Y. Self-Biased-SMA Drive PU Microgripper with Force Sensing in Visual Servo. International Journal of Advanced Robotic Systems, 10(5):9-11, 2013.

[24] F. Karimirad, S. Chauhan, and B. Shirinzadeh. Vision-based force measurement using neural networks for biological cell microinjection. Journal of Biomechanics, 47:1157-1163, 2014.

[25] P. Masa, E. Franzi, and C. Urban. Nanometric resolution absolute position encoders. page 3 pages, 2008.

[26] D.B. Boyton. Position encoder using statistically biased pseudorandom sequence, 2003. US Patent App. 10/399, 470.

[27] J. G. Zea, P. Sandoz, E. Gaiffe, J.L. Prétet, and C. Mougin. PseudoPeriodic Encryption of Extended 2-D Surfaces for High Accurate Recovery of any Random Zone by Vision. International Journal of Optomechatronics, 4(1):65-82, 2010.

[28] P. Sandoz, R. Zeggari, L. Froehly, J.L. Prétet, and C. Mougin. Position referencing in optical microscopy thanks to sample holders with out-offocus encoded patterns. Journal of Microscopy, 225(3):293-303, 2007.

[29] J. G. Zea, P. Sandoz, G. J. Laurent, L. L. Lemos, and C. Clevy. TwinScale Vernier Micro-Pattern for Visual Measurement of 1D In-Plane Absolute Displacements with Increased Range-to-Resolution Ratio . In International Symposium on Optomechatronic Technologies, volume 7 , pages 222-234, 2013.

[30] V. Guelpa, G. J. Laurent, P. Sandoz, J. G. Zea, and C. Clevy. Subpixelic Measurement of Large 1D Displacements: Principle, Processing Algorithms, Performances and Software. Sensors, 14:5056-5073, 2014. 
[31] Xide Li, Dongchuan $\mathrm{Su}$, and Zhao Zhang. A novel technique of microforce sensing and loading. Sensors and Actuators A: Physical, 153:13-23, 2009.

[32] J.A. Harley and T.W Kenny. A High-Stiffness Axial Resonant Probe for Atomic Force Microscopy. Journal of Microelectromecanical Systems, 10:434-441, 2001.

[33] Joint Committee for Guides on Metrology. International vocabulary of metrology - Basic and general concepts and associated terms (VIM), 3rd edn. 2012.

[34] T. Chen, L. Chen, B. Liu, J. Wang, and X. Li. Design of a FourArm Structure MEMS Gripper Integrated with Sidewall Force Sensor. In 4th IEEE International Conference on Nano/Micro Engineered and Molecular Systems, pages 75-79, 2009.

[35] D. A. Simson, F. Ziemann, M. Strigl, and R. Merkel. Micropipet-Based Pico Force Transducer: In Depth Analysis and Experimental Verification. Biophysical Journal, 74:2080-2088, 1998.

[36] J. Rajagopalan, A. Tofangchi, M. Taher, and A. Saif. Linear HighResolution BioMEMS Force Sensors With Large Measurement Range. Journal of Microelectromechanical Systems, 19(6):1380-1389, 2010.

[37] M. Nasir, M. Dickinson, and D. Liepmann. Multidirectional Force and Torque Sensor for Insect Flight Research. In Solid-State Sensors, Actuators and Microsystems, volume 2, pages 1625-1630, 2005.

[38] M. A. Cullinan, R. M. Panas, and Culpepper M. L. A MultiAxis MEMS Sensor with Integrated Carbon Nanotube-Based Piezoresistors for Nanonewton Level Force Metrology. Nanotechnology, 23(32):325501, 2012.

[39] R. Bharanidaran and T. Ramesh. Numerical Simulation and Experimental Investigationof a Topologically Optimized Compliant Microgripper. Sensors and Actuators A: Physical, 205:152-163, 2014.

[40] S. Sunshine, M. Balicki, X. He, K. Olds, J. U. Kang, P. Gehlbach, R. Taylor, I. Iordachita, and Handa J. T. A Force-Sensing Microsurgical Instrument that Detect Forces Below Human Tactile Sensation. National Institutes of Health, 33:200-206, 2013.

[41] Y Sun, K-T. Wan, K.P. Roberts, J. C. Bischof, and Nelson B. J. Mechanical Property Characterization of Mouse Zona Pellucida. IEEE Transactions on NanoBioscience, 2, 2003.

[42] CSM. Nanotribometer.

[43] FemtoTools. FT-S Microforce Sensing Probe.

[44] PCE Inst. Force Gauge PCE-FG 10K.

[45] PCE Inst. Precision Force Gauge PCE-FG 50

[46] Kern. Precision Balance PBS 620-3M.

[47] Timber. Weighing Platform for Vehicle ref: WWSE15T.

[48] P. Sandoz, J-C. Ravassard, S. Dembele, and A. Janex. Phase-Sensitive Vision Technique for High Accuracy Position Measurement of Moving Targets. IEEE Transactions on Instrumentation and Measurement, 49(4):867-872, 2000.

[49] J. G. Zea, Patrick Sandoz, and Laurent Robert. Position Encryption of Extended Surfaces for Subpixel Localization of Small-Sized Fields of Observation. In International Symposium on Optomechatronic Technologies, pages 22-27. IEEE, 2009.

[50] P. Moore, M. Rakotondrabe, C. Clevy, and G. Wiens. Development of a Modular and Compliant Micro-Assembly Platform with Integrated Force and Compliance Measurement Capabilities. In ICOMM 7th International Conference on MicroManufacturing, page 5 pages, 2012.

[51] N. Tan, C. Clevy, and N. Chaillet. Calibration of single-axis nanopositioning cell subjected to thermal disturbance. In IEEE International Conference on Robotics and Automation, volume 19, pages 917-922, 2013. 